Math. Model. Nat. Phenom.

Vol. 5, No. 7, 2010, pp. 109-115

DOI: $10.1051 / \mathrm{mmnp} / 20105718$

\title{
The Placement of Electronic Circuits Problem: A Neural Network Approach
}

\author{
M. Ettaouil* , K. Elmoutaouakil, Y. Ghanou \\ Scientific Computing and Computer Science, Engineering Sciences \\ Department of Mathematics and Computer Science, Faculty \\ of Science and Technology of Fez, University Sidi Mohammed ben Abdellah \\ Box 2202 Fez, Morocco
}

\begin{abstract}
The goal of this paper is to apply the Continuous Hopfield Networks $(\mathrm{CHN})$ to the Placement of Electronic Circuit Problem (PECP). This assignment problem has been expressed as Quadratic Knapsack Problem (QKP). To solve the PECP via the CHN, we choose an energy function which ensures an appropriate balance between minimization of the cost function and simultaneous satisfaction of the PECP constraints. In addition, the parameters of this function must avoid some bad local minima. Finally, some computational experiments solving the PECP are included.
\end{abstract}

Key words: placement of the electronic circuits problem (PECP), continuous Hopfield networks (CHN), quadratic Knapsack problem (QKP), combinatorial problems

AMS subject classification: $14 \mathrm{~N} 10,94 \mathrm{C} 05$

\section{Introduction}

The aim of this work is to apply the Continuous Hopfield Network (CHN) to the Placement of Electronic Circuit Problem (PECP). This problem had already been treated using the decomposition approach; see [6]. However, this method requires much CPU time. The CHN was proposed to solve any combinatorial problems; see [4]. Some authors have treated the QKP through this neuronal approach; see [3]. Within these papers, the feasibility of the equilibrium points of the CHN

*Corresponding author. E-mail: mohamedettaouil@yahoo.fr 
cannot be assured for the general case, that is the equilibrium points do not fulfill the constraints of the QKP. To avoid this problem, we choose an energy function which ensures an appropriate balance between minimization of the cost function and simultaneous satisfaction of the PECP constraints. In addition, the parameters of this function must avoid some bad local minima. Moreover, to overcome the problems observed with the Euler method, we use the new algorithm proposed in [8].

The CHN was proposed by Hopfield and Tank [4] to solve any combinatorial problems, some authors have treated the QKP through this neuronal approach [4], [5], [12]. Within these papers, the feasibility of the equilibrium points of the CHN cannot be assured for the general case, and the solutions obtained are often not good enough. To avoid these problems, we choose an appropriate energy function that penalizes the objective function and the constraints of the PECP. The penalty parameters of this function should ensure an appropriate balance between minimization of the cost function and simultaneous satisfaction of the PECP constraints. In addition, these parameters must avoid some bad local minima. To this end, we decompose the set of non feasible solutions, which do not fulfill the constraints of the PECP, into appropriate subsets, and we use the partial derivatives of the energy function to select the parameters of the function. Since the differential equation which characterizes the dynamics of the $\mathrm{CHN}$ is analytically hard to solve, many researchers have used the Euler method. However, this latter proved to be highly sensitive with respect to initial conditions, and it requires a lot of CPU time for medium or greater size CHN instances. Recently, one algorithm which assures the obtaining of the equilibrium points has been proposed. This latter is robust with respect to the initial conditions, see [11].

The paper is organized in five sections. In the second, the PECP is introduced as a Quadratic Knapsack Problem. The third section introduces the continuous Hopfield network and its application to the PECP. Finally, some computational experiments are included in the fourth section.

\section{Placement of electronic circuits problem (PECP)}

An integrated circuit consists of the central part and the border of $m$ base cells. Given $n$ cells, the PECP looks for a nonoverlaping assignment of the $n$ cells to the $m$ base cells of the central and border part, and looks for a realization of the nets such that wire ability coincides with minimum total wire length. If cells $i$ and $j$ are assigned, respectively, to the base cells $k$ and $l$, then the value $d_{i k, j l}$ denotes the distance between cells $i$ and $j$, and $C_{i j} \geq 0$ present the number of nets connecting cells $i$ and $j$. In order to take overlaps into account, the number of overlapping units $O_{i k, j l}$ is defined. The cost matrix $P$ is defined as follow

$$
P_{i k, j l}=C_{i j} d_{i k, j l}+\lambda O_{i k, j l} \forall i, j=1, \ldots, n \quad \forall k, l=1, \ldots, m,
$$

with

$$
\lambda \geq 0 .
$$

Let $\nu=\left(\nu_{1,1} \ldots \nu_{1, m} \ldots \nu_{i, 1} \ldots \nu_{i, m} \ldots \nu_{n, 1} \ldots \nu_{n, m}\right)^{t}$ be the $n \times m$ - vector of state variable such that $\nu_{i, k}=1$ if cell $i$ is assigned to base cell $k$, and $\nu_{i, k}=0$ otherwise. All cells must be assigned to exactly one of the $m$ base cells. 


$$
e_{i}(\nu) \equiv \sum_{k=1}^{m} \nu_{i, k}=1, \forall i=1, \ldots, n .
$$

The PECP problem can be formulated mathematically as follow

$$
\begin{gathered}
\operatorname{Min}\left(\frac{1}{2} \nu^{t} P \nu\right), \\
\text { Subject to : } \sum_{k=1}^{m} \nu_{i, k}=1, \forall i=1, \ldots, n, \\
\nu \in\{0,1\}^{n \times m} .
\end{gathered}
$$

This problem can be solved using the Lagrangian duality (for example, Lagrangian decompositions methods, Lagrangian substitutions etc.) [2]. But these methods are based on gradient descending technique and needs an extremely high number of iterations for training. For simplicity, the following sets are defined

$$
H_{C}=\{0,1\}^{n \times m}, H_{F}=\left\{\nu \in H_{C} / e_{i}(\nu)=1, \forall i=1, \ldots, n\right\} .
$$

\section{Continuous Hopfield network (CHN) for the PECP}

In this section we will apply the CHN to solve the PECP by choosing an appropriate energy function. The CHN of size $n$ is a completely interrelated neural network with $n$ continuous valued units. The strength of the connection from neuron $j$ to neuron $i$ is denoted by $T_{i, j}$. Let $u, \nu$ and $i^{b}$ be respectively the vectors of neuron states, outputs and biases. By considering a CHN as an electronic circuit and by applying the Kirchhoff's law and Ohm's law [1], we obtain the differential equation which governs the dynamics of $\mathrm{CHN}[1,7]$ as follows:

$$
\frac{d u}{d t}=-\frac{u}{\tau}+T \nu+i^{b}
$$

where the vector $u \in \mathbb{R}^{n}, \nu \in \mathbb{R}^{n}$, the matrix $T$ is of dimension $n \times n$ and the vector $I^{b} \in \mathbb{R}^{n}$. The coordinate $u_{i}$ of the vector $u$ and the coordinate $\nu_{i}$ of the vector $\nu$ present, respectively, the current state and the output of the neuron $\mathrm{i}$, with $\nu_{i}=g\left(u_{i}\right)$ and $g\left(u_{i}\right)=\frac{1}{2}\left(1+\tanh \left(\frac{u_{i}}{u_{0}}\right)\right)$ where $u_{0}>0$. The real values $T_{i j}$ and $I_{i}^{b}$ are, respectively, the weight of the synaptic connection from the neuron $\mathrm{j}$ to neuron $\mathrm{i}$ and the offset bias of the neuron $\mathrm{i}$. A point is called an equilibrium point of the system, if for an input vector $u_{0}, u_{e}$ satisfies $u(t)=u^{e} \forall t \geq t_{e}$ for some $t \geq 0$. Hopfield has introduced the energy function $E$ on $[0,1]^{n}$ which is defined by

$$
E(T)=-\frac{1}{2} \nu^{t} T \nu-\left(i^{b}\right)^{t} \nu-\frac{1}{\tau} \sum_{i=1}^{N} \int_{0}^{v_{i}} g^{-1}(x) d x
$$


It should be noted that if the energy function (or Layapunov function) exists, the equilibrium point exists as well. Hopfield [7] proved that the symmetry of the matrix is a sufficient condition for the existence of Lyapunov function. The $\mathrm{CHN}$ will solve combinatorial problems that can be expressed as the constrained minimization of the function:

$$
E(T)=-\frac{1}{2} \nu^{t} T \nu-\left(i^{b}\right)^{t} \nu
$$

The extrema of this function are among the corners of the -dimensional hypercube [9], [10]. The philosophy of this approach is that the objective function which characterizes the combinatorial problem is associated with the energy function of the network when $\tau \rightarrow \infty$. Given the Generalized Knapsack Quadratic Problem (GKQP):

$$
\begin{gathered}
\operatorname{Min}\left(\frac{1}{2} \nu^{t} P \nu\right)+q^{t} \nu, \\
\text { Subject to }: \\
R \nu=b, \\
\nu_{j} \in\{0,1\} \quad j=1, \ldots, m, \\
\nu_{j} \in[0,1] \quad j=m+1, \ldots, n,
\end{gathered}
$$

with the matrix $P$ of dimension $n \times n$, the vector $q \in \mathbb{R}^{n}$. The vector $\nu=\left(\nu_{1}, \ldots, \nu_{m}, \ldots, \nu_{n}\right)^{t} \in$ $I R^{n}$, the matrix $R$ is of dimension $c \times n$, and the vector $b \in \mathbb{R}^{c}$. For convenience, we adopt the following notations: $H_{d}=\{0,1\}^{m}, H_{c}=[0,1]^{c}, H=[0,1]^{n}, H_{F}=\left\{\nu \in H_{d} \times H_{c} / R \nu=b\right\}$. The standard form of the energy function is: $E(\nu)=E^{c}(\nu)+E^{p}(\nu) \forall \nu \in H$ where $E^{c}$ is directly proportional to the objective function. $E^{p}$ is a quadratic function that ensures the feasibility of the solution obtained by the $\mathrm{CHN}$, and also penalizes the violated constraints of the problem. This function must give the same value for each element $\nu$ form $H_{F}$, and an adequate selection of this function is necessary for a correct mapping. We propose the following energy function for the PECP:

$$
\begin{gathered}
E(\nu)=\frac{\alpha}{2} \sum_{i=1}^{n} \sum_{k=1}^{m} \sum_{j=1}^{n} \sum_{l=1}^{m} \nu_{i, k} p_{i k, j l} \nu_{i, k}+\frac{\Phi}{2} \sum_{j=1}^{n}\left[e_{j}(\nu)\right]^{2}+\beta \sum_{j=1}^{n} e_{j}(\nu) \\
+\gamma \sum_{i=1}^{n} \sum_{k=1}^{m} \nu_{i, k}\left(1-\nu_{i, k}\right),
\end{gathered}
$$

where $\alpha>0, \Phi \in \mathbb{R}, \beta \in \mathbb{R}, \gamma \in \mathbb{R}$. The slope $u_{0}$, to use neurons with a gradually increasing slope during the optimization process [9], and the term $\gamma \sum_{i=1}^{n} \sum_{k=1}^{m} \nu_{i, k}\left(1-\nu_{i, k}\right)$, equals to zero only if all take the binary values $\{0,1\}$, plays a crucial role in enforcing the convergence to a hypercube corner [1]. Now, we select the parameters of this function to assures the feasibility of the equilibrium point of the CHN. According to the equations (7), the weights and thresholds for the mapped PECP depend on the parameters $\alpha, \Phi, \beta$ and $\gamma$. Thus, in order to solve the PECP via the $\mathrm{CHN}$, a convenient setting of these parameters is necessary. In this part, on the basis of the 
appropriate decomposition of the set $H_{c}-H_{F}$ and on the partial derivatives of the energy function, we choose the parameters of the energy function that assures the feasibility of the equilibrium points. The partial derivatives of the generalized energy function is given by

$$
E_{i, k}(\nu)=\frac{d E}{d \nu_{i k}}=\alpha \sum_{j=1}^{n} \sum_{l=1}^{m} p_{i k, j l} \nu_{j, l}+\Phi e_{i}(\nu)+\beta+\gamma\left(1-2 \nu_{i k}\right) .
$$

For simplicity, the following parameter constraints are initially assigned. The constraint below is imposed to minimize the objective function, then $\alpha \geq 0$ The following constraint is naturally imposed to penalize the family of linear constraints: $\left\{e_{i}(\nu)=1, i=1, \ldots, n\right\}$ then $\Phi>0$. The next constraint is necessary to avoid the stability of the interior points $\nu \in H_{c}-H_{F}: T_{i k, i k}=$ $-\Phi+2 \gamma \geq 0$. Given the family of constraints of PECP: $e_{i}(\nu)=1, \forall i \in\{1, \ldots, n\}$. The partition of $H_{c}-H_{F}$ is defined as: $U_{1}=\left\{\nu \in H_{c} / \exists i, e_{i}(\nu)<1\right\} \bigcap\left\{\nu \in H / \forall j, e_{j}(\nu) \leq 1\right\}$. In this case, a non-assigned cell $i \in\{1, \ldots, n\}$ exists such that $\nu_{i k}=0 \forall k=1, \ldots, m$, and thus the value $\nu_{i k}$ will increase if $E_{i k} \leq-\varepsilon$. Now the following constraint is obtained:

$$
E_{i k} \leq \alpha p_{\max } n+\beta+\gamma \leq-\varepsilon
$$

where $p_{\max }=\operatorname{Max}\left\{p_{i k, j l}\right\}, U_{2}=\left\{\nu \in H_{c} / \exists i, e_{i}(\nu)>1\right\}$, in this case, one cell $i \in\{1, \ldots, n\}$ is assigned to two base cells $k \neq l$ so that $\nu_{i, k}=\nu_{i, l}=1$ and therefore the value $\nu_{i, k}$ will decrease if $E_{i, k} \geq \varepsilon$. The following constraint is obtained: $E_{i k} \leq \alpha p_{\min }+2 \Phi+\beta-\gamma \geq-\varepsilon$, where $p_{\text {min }}=\operatorname{Min}\left\{p_{i k, j l}\right\}$. Moreover, the solution can be feasible by choosing:

$$
\gamma=\frac{\alpha\left(n p_{\max }-p_{\min }\right)+2 \varepsilon}{2}, \quad \Phi=2 \xi, \quad \beta=-\frac{4 \xi+\alpha\left(n p_{\max }+p_{\min }\right)}{2},
$$

this parameter-setting depends on the parameters $\varepsilon, \alpha$ the number of base cells $m$, the number of cells $n$ and on the matrix $\mathrm{P}$.

\section{Computational experiments}

To simulate the mapped PECP we use the algorithm described in [8], with the values of parameters $\alpha=\frac{1}{n * m}, m=16$ and $\varepsilon=0,01$. In addition, the data of the PECP and the initial states were randomly generated. The results of the simulation reported in Table 1 are obtained on pentium III $666 \mathrm{MHz}$ through Borland $\mathrm{C}++$ Builder 3. The Table 1 shows, respectively, the number of cells, the density of circuit, the overlaps option, the number of simulations, the mean wiring length and the mean of CPU time. Finally, all CHN simulations produces valid placement, this means that each cell is assigned to exactly one of the $m$ base cells. The CHN method (Table 1) performs much better than the decomposition method with respect to the mean estimated wiring length, and the CPU time [8]. 


\begin{tabular}{|c|c|c|c|c|}
\hline the num. of cells & den. of circuit & num. of run & mean wir. length & mean time (s) \\
\hline 1022 & 50 & 20 & 54132.5 & 945.7 \\
\hline 2293 & 23.6 & 15 & 77473.8 & 1719.1 \\
\hline 2670 & 50.3 & 10 & 93762.13 & 1900.4 \\
\hline
\end{tabular}

Table 1: Computational results of the PECP instances by $\mathrm{CHN}$ method

\section{Conclusion}

In this work, we have applied the CHN to solve the PECP by choosing an appropriate energy function. This choice ensures that the solutions obtained are always feasible. To make this approach more efficient, it can be combined with other metaheuristics [3], or it can be computationally optimized by introducing analytical improvements, such as replacing the hyperbolic tangent, with a linear function.

\section{Acknowledgements}

The authors would like to express their sincere thanks for the referee for her/his helpful suggestions.

\section{References}

[1] A. Cichocki, R. Unbehauen. Neural networks for optimization and signal processing. B.G. Teubner Stuttgart, 1993.

[2] M. Ettaouil. Contribution à l'étude des problèmes de satisfaction de contraintes et à la programmation quadratiques en nombre entiers, allocation statiques de tâches dans les systèmes distrubués, thèse d'état, Université Sidi Mohammed ben Abdellah, F.S.T. de Fès, 1999.

[3] M. Ettaouil, Y. Ghanou. Neural architectures optimization and Genetic algorithms. Wesas Trasactions on Computer, 3 (2009), No. 8, 526-537.

[4] A.H. Gee, S.V.B. Aiyer, R.W. Prager. An analytical framework for optimizing neural networks. Neural Networks, (1993), No. 6, 79-97.

[5] A. Ghosh, S.K. Pal. Object Background classification using Hopfield type neural networks. International Journal of Patten Recognition and Artificial Intelligence, (1992), No. 6, 9891008 .

[6] J.J. Hopfield, D.W. Tank. Neural computation of decisions in optimisation problems. Biological Cybernetics, 52 (1985), 1-25. 
[7] J.J. Hopfield. Neurons with graded response have collective computational properties like those of two-states neurons. Proceedings of the National Academy of Sciences of USA, 81 (1984), 3088-3092.

[8] M. Jünger, A. Martin, G. Reinel, R. Weismantel. Quadratic 0/1 optimization and a decomposition approach for the placement of electronic circuits. Report 91. 102, Institut für Informatik Universität köln, (1991).

[9] B.W. Lee, B.J. Shen. Hardware annealing in electronic neural networks. IEEE Trans, 1 (1990), $134-137$.

[10] N.M. Nasrabadi, C.Y. Choo. Hopfield network for stereo vision correspondence, New York: Marcel Dekker, 1994.

[11] P.M. Talavàn, J. Yànez. A continuous Hopfield network equilibrium points algorithm. Computers and Operations Research, 32 (2005), 2179-2196.

[12] K. Tatsumi, Y. Yagi, T. Tanino. Improved projection Hopfield network for the quadratic assignment problem. SICE 2002, proceedings of the 41 st SICE annual conference, 4 (2002), 2295-2300. 